\title{
Changing trends in intracranial abscesses secondary to ear and sinus disease
}

\author{
S TANDON, N BEASLEY*, A C SwiFT
}

\begin{abstract}
Objectives: To review the management, causative organisms, morbidity and mortality of intracranial abscesses secondary to sinus and ear disease.

Study design and setting: Retrospective, case note review of suppurative intracranial complications of ear and sinus disease in patients admitted to a regional neurosurgical centre between 1980 and 2004. These data were compared with published material from the same region from 1950-1979.

Results: There was a marked reduction in the mortality rate and the number of intracranial abscesses secondary to chronic ear disease, comparing the two time periods. However, there was little change in the percentage of sinus-related abscesses treated and in their symptoms, signs, abscess location and long term morbidity. Microbiology results showed that streptococcal species predominated as causative organisms, with a high percentage of anaerobic bacteria in otogenic abscesses.

Conclusion: Despite improved outcomes, a high index of suspicion for intracranial complications of ear or sinus disease should be maintained in the presence of appropriate signs and symptoms.
\end{abstract}

Key words: Intracranial Abscess; Ear; Para-Nasal Sinus; Trends

\section{Introduction}

Acute and chronic sinus and ear disease can give rise to a spectrum of complications. Of these, intracranial problems are potentially the most serious. This group of complications includes suppurative disease (such as intracerebral abscess and sub- or extradural collection) and non-suppurative complications (such as meningitis, lateral sinus thrombosis, cavernous sinus thrombosis and otitic hydrocephalus). ${ }^{1,2}$ These complications carry with them a risk of mortality and significant morbidity.

In light of these significant and important problems, we retrospectively reviewed the patients admitted to our regional neurosurgical unit with intracranial abscesses secondary to ear and nasal disease, over a 24-year period. From this data, we reviewed patient management, common causative organisms, and morbidity and mortality figures. We then compared these findings with the results of a similar review from our region which assessed an earlier time period, analysing any changes in trends, management and outcomes.

\section{Materials and methods}

A retrospective review of records of patients with a coding of brain abscess admitted to the Walton
Centre for Neurology and Neurosurgery, Liverpool, UK, between 1 January 1980 and 31 December 2004 was undertaken. Patients were identified from hospital, theatre and post-mortem records. Inclusion criteria were patients with a diagnosis of an intracranial abscess (including sub- and extradural collections) secondary to otological or rhinological disease. These cases were identified as being otogenic or rhinogenic if there was clear evidence from the medical records of this being the case. Specific details from the history, examination or investigations consistent with an otogenic or rhinogenic origin were sought. Brain abscesses from other sources were excluded.

The following data were collected: demographics; presenting symptoms and signs (of ENT source and brain abscess); aetiology of abscess; history of previous nasal or ear disease; microbiology samples and results; and medical and surgical management of both the abscess and the primary source of infection. Morbidity and mortality data were also obtained. These data were obtained by review of case notes, drug charts, operation notes and microbiology results. Results were collated onto a Microsoft Excel worksheet and categorised according to the primary infective source.

From the Department of Otolaryngology, Head and Neck Surgery, Aintree University Hospitals NHS Foundation Trust, Liverpool, and the *Department of Otolaryngology, Head and Neck Surgery, Nottingham University Hospitals NHS Trust, Queen's Medical Centre, Nottingham, UK.

Accepted for publication: 19 February 2008. First published online 19 May 2008. 
Data collected for the current study were compared to the results from two similar studies performed in the same geographical region between 1950-1979.3,4 Specific comparisons were made between the source and site of the abscess, morbidity and mortality data. Data collection for these studies was performed via theatre or post-mortem records. ${ }^{3,4}$

\section{Results}

Two hundred and twenty-eight patients with a coding of brain abscess were admitted to the Walton Centre for Neurology and Neurosurgery, Liverpool, UK, between 1 January 1980 and 31 December 2004. Of these 228 patients, notes were obtained for 196 cases. Fifty-three patients were suitable for inclusion.

All patients underwent computed tomography (CT) scanning of the head. Patients with suspected infective processes were initially treated medically with broad spectrum antibiotics using various regimes that are detailed later. Antimicrobial therapy was changed if appropriate after microbiological results and discussion with the medical microbiologist.

\section{Combined group}

Of all the intracranial abscesses treated at our institution over the 24-year study period, otogenic abscesses accounted for 9.7 per cent and rhinogenic abscesses for 14.6 per cent. In the 53 cases identified, there was a preponderance of men (male to female ratio, 4:1). Age at presentation ranged from four to 76 years (median age, 29 years). The demographics of the non-ENT source brain abscesses show a male to female ratio of $2.5: 1$ with an age range of 4 months to 74 years (mean age 38 years).

Twenty-two cases were secondary to ear disease and 31 to sinus disease. Five of the 53 patients died as a direct result of the infection: three cases from ear pathology and two from sinus pathology. Admission trends over the study period showed no obvious pattern for either the otological or rhinological groups (Figure 1). Headache and altered consciousness were the predominant neurological symptoms for both the otogenic and rhinogenic groups (Table I).

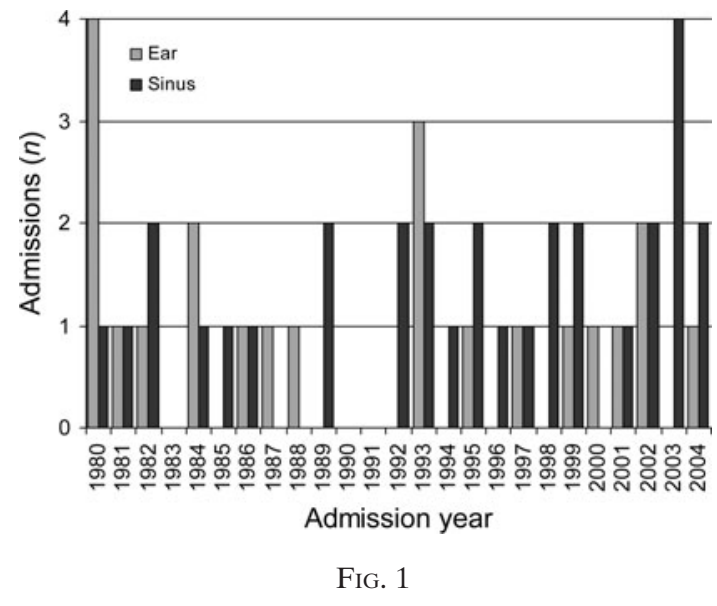

Intracranial abscess admissions by year and source.
TABLE I

PRESENTING SYMPTOMS AND SIGNS

\begin{tabular}{|c|c|c|}
\hline Symptom or sign & 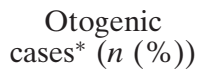 & $\begin{array}{c}\text { Rhinogenic } \\
\operatorname{cases}^{\dagger}(n(\%))\end{array}$ \\
\hline \multicolumn{3}{|l|}{ Neurological } \\
\hline Headache & $16(73)$ & $23(74)$ \\
\hline Altered consciousness & $14(64)$ & $19(61)$ \\
\hline Neck stiffness & $10(45)$ & $9(29)$ \\
\hline Photophobia & $10(45)$ & $5(16)$ \\
\hline Ataxia & $9(41)$ & $5(16)$ \\
\hline Pyrexia & $8(36)$ & $12(39)$ \\
\hline Vomiting & $8(36)$ & $11(35)$ \\
\hline Nausea & $6(27)$ & $10(32)$ \\
\hline Limb dysfunction & $5(23)$ & $9(29)$ \\
\hline Cranial nerve abnormalities & $4(18)$ & $6(19)^{*}$ \\
\hline Dysphasia & $4(18)$ & $5(16)$ \\
\hline Confusion & $3(14)$ & $10(32)$ \\
\hline Seizures & $3(14)$ & $7(23)$ \\
\hline Coryzal symptoms & $2(9)$ & $3(10)$ \\
\hline Collapse & $2(9)$ & $4(13)$ \\
\hline Personality change & 0 & $4(13)$ \\
\hline Respiratory arrest & $1(4.5)$ & 0 \\
\hline Incontinence & 0 & $1(3)$ \\
\hline \multicolumn{3}{|l|}{ Otological } \\
\hline Otorrhoea & $13(59)$ & \\
\hline Otalgia & $11(50)$ & \\
\hline Vertigo or dizziness & 4 (18) & \\
\hline \multicolumn{3}{|l|}{ Rhinological } \\
\hline Rhinorrhoea & & $13(42)$ \\
\hline Sinus pain & & $13(42)$ \\
\hline Nasal blockage & & $10(32)$ \\
\hline Periorbital cellulitis & & $5(16)$ \\
\hline
\end{tabular}

${ }^{*} n=22 ;{ }^{\dagger} n=31 .{ }^{\ddagger}$ All visual.

\section{Otological cases}

Of the 22 patients with abscess secondary to otological pathology, six had acute otitis media (AOM), two had acute mastoiditis and 12 had chronic suppurative otitis media (CSOM) of whom seven had cholesteatoma. Three patients had undergone previous mastoid surgery (five days, four years and 10 years before admission to the neurosurgical unit). Two patients presented with otalgia and a discharging ear accompanying their cranial symptoms, but there were no further details specific to the presenting ear disease identifiable from the case notes. Otorrhoea and otalgia were present in 59 per cent and 50 per cent of patients, respectively (Table I). Fourteen patients (64 per cent) had abscesses in the cerebellum $(n=7)$ or temporal lobe $(n=7)$ (Table II).

Microbiology samples were obtained in all 22 patients. These included: pus from the brain abscess plus an ear swab (19 patients); an ear swab only (two patients); and a cerebrospinal fluid (CSF) sample plus intracranial pus (one patient). Sixteen of the 22 (77 per cent) patients had a positive culture and six had 'no growth'; of the latter, three patients had bacteria identified microscopically.

Cultures identified a single causative organism in 16 patients; three cases were polymicrobial. The predominant organisms were streptococcal species and anaerobes (Table III).

All patients were treated with antibiotics, but the specific therapy was not recorded for two patients. Most patients were treated with metronidazole combined with a broad spectrum antibiotic, with 
TABLE II

LOCATION OF INTRACRANIAL ABSCESS

\begin{tabular}{lcc}
\hline Site & $\begin{array}{c}\text { Otogenic } \\
\text { cases* }^{*}(n)\end{array}$ & $\begin{array}{c}\text { Rhinogenic } \\
\text { cases }^{\dagger}(n)\end{array}$ \\
\hline Cerebral & 19 & 16 \\
Cerebellar & 7 & 0 \\
Temporal & 7 & 1 \\
Frontal & 0 & 11 \\
Fronto-parietal & 1 & 1 \\
Parietal & 1 & 0 \\
Parieto-occipital & 1 & 0 \\
Occipital & 1 & 0 \\
Multiple & 1 & 3 \\
Subdural & 2 & 13 \\
Extradural & 1 & 2 \\
\hline
\end{tabular}

${ }^{*} n=22 ;{ }^{\dagger} n=31$.

a preference for cephalosporins. There was no preferred treatment regime.

Nine patients were treated with intravenous antibiotics alone. Adjunctive interventional treatment included: a burr hole or craniotomy $(n=19)$; stereotactic aspiration $(n=2)$; unspecified abscess aspiration $(n=1)$; mastoid exploration $(n=12)$; and mastoid cavity suction clearance $(n=1)$.

Thirteen of the 22 patients (62 per cent) had neurological sequelae (Table IV); the remaining six had no further problems after treatment.

\section{Rhinological cases}

Of the 31 cases secondary to rhinological pathology, all were due to acute sinusitis. The commonest sinus affected was the frontal, but details of the degree and location of sinus disease had not been recorded for the majority of patients (Table V). Coexisting sinus morbidity included periorbital cellulites $(n=5)$, polypoidal chronic rhinosinusitis $(n=3)$, chronic rhinosinusitis $(n=2)$ and frontal sinus osteoma $(n=1)$.

TABLE III

MICROBIOLOGY RESULTS

\begin{tabular}{lcc}
\hline Organism & $\begin{array}{c}\text { Otogenic } \\
\text { cases* }^{*}(n)\end{array}$ & $\begin{array}{c}\text { Rhinogenic } \\
\text { cases }^{\dagger}(n)\end{array}$ \\
\hline Aerobes & & \\
Pneumococcus & 3 & 4 \\
Streptococcus sp & 2 & 6 \\
Streptococcus milleri & 0 & 1 \\
Haemolytic streptococcus & & \\
$\quad$ group C & 1 & 4 \\
Haemophilus influenzae & 0 & 2 \\
MSSA & 3 & \\
Coagulase-negative & & 2 \\
$\quad$ staphylococcus & & 1 \\
Anaerobes & 1 & 1 \\
Anaerobes & 1 & 0 \\
Bacteriodes sp & 0 & 0 \\
Fusobacterium nucleatum & & \\
Facultative anaerobes & 2 & \\
Poliforms & 1 & \\
\hline
\end{tabular}

${ }^{*} n=22 ;{ }^{\dagger} n=31$. MSSA $=$ methicillin-sensitive Staphylococcus aureus
TABLE IV

MORBIDITY AND MORTALITY

\begin{tabular}{|c|c|c|}
\hline Outcome & $\begin{array}{l}\text { Otogenic } \\
\text { cases* }^{*}(n)\end{array}$ & $\begin{array}{l}\text { Rhinogenic } \\
\text { cases }^{\dagger}(n)\end{array}$ \\
\hline Morbidity & $13(59 \%)$ & $13(42 \%)$ \\
\hline Seizures & 5 & 6 \\
\hline Limb or gait problems & 4 & 3 \\
\hline Visual change & 2 & 0 \\
\hline Hearing loss & 1 & 0 \\
\hline Facial nerve weakness & 1 & 0 \\
\hline Headaches & 0 & 2 \\
\hline Aphasia & 0 & 1 \\
\hline Tracheostomy (long term) & 0 & 1 \\
\hline Mortality & 3 & 2 \\
\hline
\end{tabular}

${ }^{*} n=22 ;{ }^{\dagger} n=31$.

Rhinorrhoea and sinus pain were the commonest ENT symptoms and were each present in 42 per cent of patients (Table I). Seventy-seven per cent of abscesses in the sinus group were subdural or frontal (Table II).

Microbiology samples were obtained in 30 of the 31 patients. These included pus from the brain abscesses $(n=30)$, antral washout fluid $(n=22)$, frontal trephine aspirates $(n=2)$, nasal swabs $(n=2)$, blood $(n=2)$ and CSF $(n=2)$. Of these (total sinus patients $(n=31)), 21 / 31$ (68 per cent) of patients had a positive culture result. Of the 10 patients with no growth on culture, one had bacteria identified microscopically. Cultures identified a single causative organism on 20 occasions; two cases were polymicrobial. The predominant organisms were streptococcal species and haemophilus species (Table III).

All patients received antibiotic therapy, but the regimen was not specified for two patients. As with the otogenic group, the majority of patients were treated with metronidazole combined with broad spectrum cover (predominantly a cephalosporin). There was a preference for the use of penicillin, chloramphenicol and metronidazole in the rhinogenic group.

Interventional treatment included: a burr hole or craniotomy ( $n=30$; 97 per cent); stereotactic aspiration $(n=1)$; antral washout $(n=23)$; external frontal sinus drainage $(n=8)$; and endoscopic sinus surgery $(n=5)$. Eleven patients received topical nasal decongestants.

TABLE V

AFFECTED SINUS IN RHINOGENIC CASES

\begin{tabular}{ll}
\hline Sinus & Cases* $^{*}(n)$ \\
\hline Pansinusitis & 3 \\
Frontal & 2 \\
Frontal \& maxillary & 1 \\
Frontal sinus osteoma & 1 \\
Ethmoid \& sphenoid & 1 \\
Maxillary & 1 \\
Not specified & 22 \\
\hline
\end{tabular}

${ }^{*} n=31$. 
Sixteen of the 31 patients had no sequelae, but 13 (42 per cent) had further problems after treatment (Table IV).

\section{Comparison with clinical data from 1950-1979}

Two previous studies, published between 1950 and 1979, had assessed intracranial abscesses of otogenic or sinus origin within our region. ${ }^{3,4}$ These papers by Bradley et al. allow us to analyse changes in the disease and its outcomes between the two time periods (Table VI).

The first point to note is that the percentage of abscesses secondary to ear disease had dropped dramatically, from $24.8^{3}$ to 9.7 per cent, with the percentage of CSOM cases also reducing (from 98 per cent in $1950-79^{3}$ to 55 per cent currently). This may have been due to the increased general use of antibiotics for discharging ears in primary care, as well as prompt referral to ENT services for management of CSOM. The incidence of sinus-related abscesses had essentially remained unchanged (15.7 per cent ${ }^{4}$ compared with 14.6 per cent currently).

The symptoms and signs recorded during the two time periods showed only slight differences, as would be expected. Importantly, the commonest single symptom for all groups was headache; alteration in consciousness, vomiting, neck stiffness and pyrexia were also frequent features of all causes in both time periods.

Regarding abscess location, otogenic cases showed a reduction in temporal lobe collections, from $60^{3}$ to 32 per cent, but no change in cerebellar abscess frequency. Rhinogenic cases also showed no change in frequency of location of the collection, comparing the earlier study and our own; the commonest location was still subdural, followed by frontal lobe.

TABLE VI

DATA COMPARISON: $1950-1979^{3,4}$ VS 1980-2004

\begin{tabular}{|c|c|c|}
\hline Parameter & $1950-1979^{3,4}(\%)$ & $1980-2004(\%)$ \\
\hline \multicolumn{3}{|l|}{ Aetiology } \\
\hline Otogenic* & 24.8 & 9.7 \\
\hline CSOM & 98 & 55 \\
\hline Acute ear disease & 2 & 36 \\
\hline Rhinogenic* & 15.7 & 14.6 \\
\hline \multicolumn{3}{|l|}{ Abscess site } \\
\hline Temporal & 60 & 32 \\
\hline Cerebellar & 32 & 32 \\
\hline Subdural & 48 & 42 \\
\hline Frontal & 37 & 36 \\
\hline \multicolumn{3}{|l|}{ Morbidity } \\
\hline \multicolumn{3}{|l|}{ - Otogenic } \\
\hline - Epilepsy & 23 & 23 \\
\hline - Visual disturbance & 10.7 & 9 \\
\hline - Limb dysfunction & 12 & 18 \\
\hline \multicolumn{3}{|l|}{ - Rhinogenic } \\
\hline - Epilepsy & 35 & 19 \\
\hline - Limb dysfunction & 2 & 10 \\
\hline \multicolumn{3}{|l|}{ Mortality } \\
\hline Otogenic & 47.2 & 5.7 \\
\hline Rhinogenic & 25.9 & 3.8 \\
\hline
\end{tabular}

*Figure represents percentage of total intracranial abscess admissions. $\mathrm{CSOM}=$ chronic suppurative otitis media
Morbidity for ear disease related intracerebral abscess changed very little between 1950-79 and 1980-2004. There was no difference in the percentage of patients having epilepsy or visual disturbance; however, there was a small increase in patients with limb dysfunction. In sinus disease related abscess, however, epilepsy rates fell from $35^{4}$ to 19 per cent; again, limb dysfunction increased from $2^{4}$ to 10 per cent.

Mortality figures showed a dramatic improvement. The mortality rate for otogenic cases was 47.2 per cent $^{3}$ in the 1950-79 group and 5.7 per cent in the current group. The sinus group had a mortality rate of 25.9 per cent in $1950-79^{4}$ and 3.8 per cent in the current group. This trend has also been seen in a number of other centres over time. The reasons for this trend may well include earlier diagnosis of intracranial complications due to ubiquitous CT scanning, use of third generation cephalosporins and metronidazole, advances in intensive care, and early surgical intervention. 5,6

\section{Discussion}

The brain is an organ which by virtue of its anatomy is relatively impervious to infection. The combination of the tight blood brain barrier, good blood supply and meninges results in low rates of infection. However, should a pathogen cause an infectious process within the cranium, the brain responds in a recognised fashion. The first effect is a localised cerebritis, which ultimately forms an encapsulated collection. The majority of brain abscesses occur as a result of either haematogenous metastatic seeding of microbes, via direct extension or as a complication of meningitis; up to 30 per cent of cases have no obvious identifiable source.

Intracranial abscesses typically present with a poorly localised, dull headache. As the infection progresses, symptoms and signs of raised intracranial pressure typically develop, less commonly accompanied by symptoms and signs of sepsis. Focal neurological signs are dependent on the location of the collection. For example, in our series, all the cranial nerves affected in the rhinogenic cases were visual. Management of intracranial abscess consists of adequate history and examination coupled with appropriate investigations, namely, imaging studies in the form of $\mathrm{CT}$ and magnetic resonance imaging. Early and prompt neurosurgical input should result in drainage or aspiration of the collection and, importantly, obtaining material for microbiological culture and sensitivity.

An opinion from an otolaryngologist is as essential as that of the neurosurgeon as it enables eradication of the primary focus of infection and identification of the causative organism. In the otogenic cases, mastoidectomy was done in 12 patients: two with acute mastoiditis; two with AOM; two with CSOM and six with cholesteatoma. One patient with recurrent cholesteatoma within a mastoid cavity underwent suction clearance. Mastoidectomy was performed one week before neurosurgery in one patient with acute mastoiditis; at the same time as neurosurgery in nine cases; and within two weeks of neurosurgery in 
two, where referral had been deferred until after craniotomy or aspiration.

In the rhinogenic group, 28 of 31 patients underwent an ENT surgical intervention, such as maxillary sinus washout and/or external frontal sinus drainage. This was done under the same anaesthetic as the neurosurgical intervention in 10 patients. The remaining 18 patients underwent sinus drainage at a later date because of lack of improvement with conservative treatment, or following drainage of the intracranial collection.

Intracranial infections arising from the middle ear or paranasal sinuses have a well recognised pathway of spread - this may be directly or by septic emboli. CSOM with cholesteatoma may spread via direct extension, as a result of erosion of bone, towards the tegmen and so the middle cranial fossa dura. This results in the majority of collections being located in the cerebellar or temporal regions - as found in our study. The majority of the otogenic patients in our study had a history of CSOM with cholesteatoma. The risk of brain abscess in these patients is estimated as one in 10000 , with a lifetime risk over 30 years of one in $200 .^{7}$ It should be borne in mind that the old concept of 'safe' CSOM (i.e. mucosal disease without cholesteatoma) does not reduce the risk of development of complications. In a retrospective study, Browning found that 38 per cent of brain abscesses secondary to chronic ear disease were from 'safe ears'. Furthermore, 15 per cent of the patients in Browning series had previously undergone modified radical mastoid exploration - surgery designed to eradicate disease and prevent complications.

The complex, valveless venous system of the sinuses provides a route for septic thrombus to enter the intracranial system. The anatomical relation of the sinuses to the brain, especially the frontal sinus, allows direct spread. Direct extension may also occur in patients with anatomical defects between the sinuses and the cranium; these may be congenital, surgical or traumatic. The importance of the frontal sinus is highlighted in our series; this sinus was affected in the vast majority of rhinogenic cases. This in turn causes a collection in the frontal lobe or subdural space, as seen in the majority of our rhinogenic cases.

Similar to previous reported findings, ${ }^{9}$ most of our rhinogenic abscesses had acute sinusitis but no history of sinus disease - only 18 per cent had preexisting sinus disease. However, the converse is true for otogenic cerebral abscesses. This is the likely reason for the current predominance of rhinogenic abscesses compared with those of otogenic origin prevention of acute sinusitis is nigh on impossible, whereas prevention and eradication of chronic ear disease has improved over the years.

The bacteriology results of our cases show, for both ear and nasal cases, the predominance of pure cultures over mixed cultures, with streptococcal species being the commonest pathogenic organisms. This is to be expected in light of the fact that these organisms will commonly give rise to primary infections in the middle ear and paranasal sinuses. Interestingly, anaerobic bacteria were the second commonest group found in the otogenic cases. This was also found in a multi-centred prospective study by de Louvois et al. ${ }^{10}$ who observed that anaerobes were principally isolated from temporal lobe abscesses from an ear source. These authors reported growth from 100 per cent of the brain abscess samples they cultured, compared with 14-29 per cent in our series. They commented that prompt inoculation and incubation of samples (with standard microbiological techniques) must be performed in order to increase a positive culture yield, and that this should be performed irrespective of the time of day.

- The percentage of intracranial abscesses secondary to ear disease reduced over time, as did that of abscesses secondary to chronic serous otitis media. The percentage of rhinogenic cases remained effectively the same

- Abscess location was predominantly temporal lobe and cerebellar in otogenic cases, and predominantly subdural and frontal lobe in rhinogenic cases

- Mortality rates from intracranial complications of ear and sinus disease improved over time; however, morbidity rates changed only minimally

- Streptococcal species are the predominant causative organism for rhinogenic and otogenic intracranial collections. Anaerobes were commonly found in otogenic cases

Our intention in comparing our current findings with those reported between 1950-1979 is to review recent trends and identify potential changes in the aetiology and outcome of this significant infective condition. We acknowledge that there are inherent limitations in making a strict comparison between time periods such as major advances in disease management, as well as changes in the archiving of medical records.

\section{Conclusions}

Overall, the percentage of ENT mediated brain abscesses in our region has decreased over time. Rhinogenic abscesses are more common than otogenic abscesses due to marked reduction in chronic ear disease (CSOM). There has been no significant change in presentation or intracranial location of the collection related to the primary source. The types of long term complications for both ear and nasal cases are similar with more problems seen in the otogenic group. There is a marked reduction in mortality which may well due to advances in antimicrobial management, intensive care and imaging.

We recommend antimicrobial cover for streptococcal species with inclusion of metronidazole in the treatment regime. Mastoidectomy is indicated in the presence of mastoiditis or CSOM especially with cholesteatoma. For suppurative sinus disease, patients should undergo maxillary antral washout 
and frontal sinus trephination. In the ideal situation, operative ENT management should be performed in the same sitting as neurosurgical intervention; this decreases anaesthetic morbidity as well as tackling the source of the infection at the same time as the intracranial complication. Finally we would recommend that the sinuses and the middle ear cleft are included in any scans of the brain to identify changes indicative of an ear or nasal source of the intracranial collection.

\section{Acknowledgements}

The authors would like to thank Mr Patrick Foy, Consultant Neurosurgeon, and Dr Richard Cooke, Consultant Microbiologist, for their help and advice with this paper.

\section{References}

1 Wright JL, Grimaldi PM. Otogenic intracranial complications. J Laryngol Otol 1973;87:1085-96

2 Gallagher RM, Gross CW, Phillips CD. Suppurative intracranial complications of sinusitis. Laryngoscope 1998;108: $1635-42$

3 Bradley PJ, Manning KP, Shaw MD. Brain abscess secondary to otitis media. J Laryngol Otol 1984;98:1185-91

4 Bradley PJ, Manning KP, Shaw MD. Brain abscess secondary to paranasal sinusitis. J Laryngol Otol 1984;98:719-25
5 Keet PC. Cranial intradural abscess management of 641 patients during the 35 years from 1952 to 1986 . Br J Neurosurg 1990;4:273-8

6 Alderson D, Strong AJ, Ingham HR, Selkon JB. Fifteenyear review of the mortality of brain abscess. Neurosurgery 1981;8:1-6

7 Nunez DA, Browning GG. Risks of developing an otogenic intracranial abscess. J Laryngol Otol 1990;104: $468-72$

8 Browning GG. The unsafeness of 'safe' ears. J Laryngol Otol 1984;98:23-6

9 Jones NS, Walker JL, Bassi S, Jones T, Punt J. The intracranial complications of rhinosinusitis: can they be prevented? Laryngoscope 2002;112:59-63

10 de Louvois J, Gortvai P, Hurley R. Antibiotic treatment of abscesses of the central nervous system. Br Med J 1977;2: $985-7$

Address for correspondence:

Sankalap Tandon,

Department of ENT,

University Hospital Aintree,

Lower Lane,

Liverpool L9 7AL, UK.

Fax: 01515295263

E-mail: sank.tandon@gmail.com

Mr S Tandon takes responsibility for the integrity of the content of the paper.

Competing interests: None declared 